\title{
Anionic Gold
}

\author{
Joseph J. Lagowski
}

Department of Chemistry, The University of Texas, Austin, U.S.A.

In recent years, research into the formation and properties of metallic anions in solution has led to interesting advances in our knowledge of alkali metal and gold anions. The latter may have possible uses in the separation of gold from base metals and in refining applications.

Until recently, the dogma of chemistry insisted that the chemistry of metals was dictated by their ability to form cations (positively charged species). This was in accordance with the fact that the chemistry of active metals such as sodium, as well as that of the noble metals, seemed to be dominated by the tendency of such elements to form cations, and involved extensively the reactions of cationic species.

In the case of gold, a survey of the literature shows that the important oxidation states of the metal are $\mathrm{Au}(\mathrm{I}), \mathrm{Au}(\mathrm{II})$ and $\mathrm{Au}(\mathrm{III})$ and it is these species, or their derivatives, that are involved in the chemistry of gold recovery and/or refining.

Recent studies have indicated, however, that certain metals can also give rise to anionic (negatively charged) species. They have suggested that there may be an untapped potential for establishing a new branch of chemistry in which the feasibility of metals forming anionic species could be explored. This potential has been realized for most of the alkali metals (sodium, potassium, rubidium and cesium) and for gold. Although these metals have been shown to form anionic species, the chemistry of such species and the technology deriving from it are virtually unexplored.

\section{Anion Formation}

From a thermodynamic point of view, the energetics of anion formation can be described by the cycle shown in Figure 1 . The energy represented by $E_{4}$ is a measure of the relative stability of the anion and, relative to the standard potential in the solvent of interest, is the parameter of interest in a study of metal anions. It may be considered as a guide to the systems which we might be able to investigate. In Figure 1, $E_{1}$ is the sublimation energy of the metal, $E_{2}$ is the electron affinity and $E_{3}$ is the solvation energy of the anion in the solvent of interest. Using the principle of conservation of energy for cycles of this type, $E_{4}$ could be obtained from the summation of these other energies:

$$
E_{4}=E_{1}+E_{2}+E_{3}
$$

Unfortunately, there are insufficient basic data to establish $E_{4}$ values unambiguously for different solvent systems. However, a qualitative assessment of the relative stabilities of anionic species can be obtained from a consideration of the energy

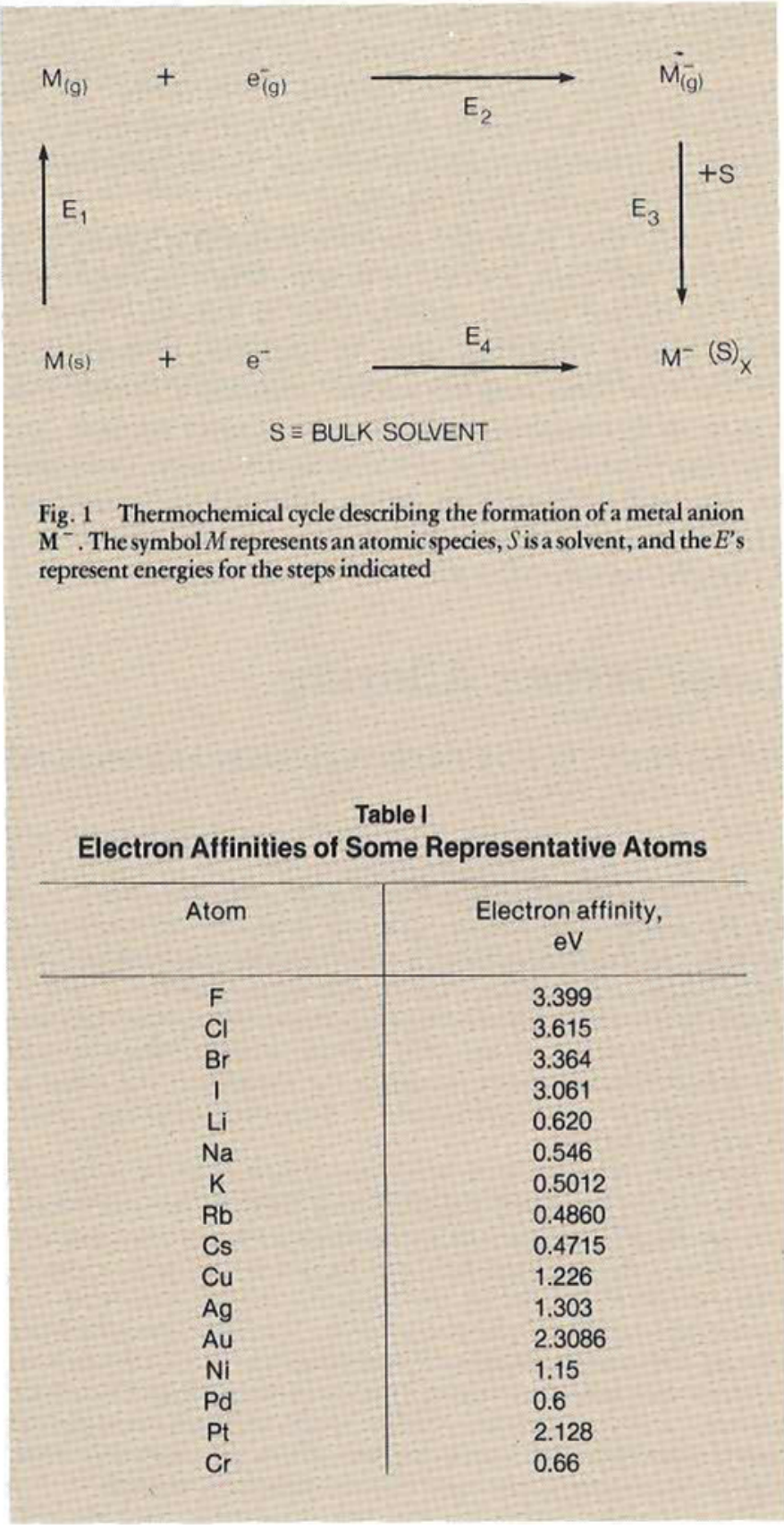

Gold Bull., 1983, 16,(1) 
required to form anions from the atoms (the electron affinity $E_{2}$ ) and of the solvation energy of the anions $E_{3}$. This semiqualitative approach ignores the contribution of the sublimation energy $E_{1}$ which could be of significance in certain instances.

The electron affinities for some representative elements are given in Table I. The electron affinity of an atom is so defined that a positive value indicates that the anion is more stable than the corresponding neutral atom. The electron affinities of the halogen atoms are given in Table I for reference purposes, because the stabilities of the halide ions are well known. The data in Table I suggest that some metallic elements form stable anions in the gaseous phase. Indeed, inspection of a complete table of electron affinities (1) shows that all the metallic elements have positive electron affinities. The problem is that of the stabilization of metal anions in a solvent. This leads to a consideration of the second major factor in our qualitative arguments based on the energy relationships displayed in Figure 1, namely the solvation energy.

Because of the theoretical difficulty of establishing even relative values for the solvation energies of ions in a given solvent, the magnitude of the solvation energy is, perhaps, the weakest link in the stability arguments based upon the cycle presented in Figure 1. Sufficient data are available, however, to give an insight into the relative magnitude of the energies involved. The solvation energies of several ions in water are listed in Table II. Two important points emerge from the data. First, the solvation energies contribute to the stabilities of all ionic species when these are dissolved in water. Secondly, the solvation energies of both cations and anions are essentially of the same magnitude. Although most chemists have an intuitive feel for the ready solvation of cations, they are often surprised to learn that anions can be as strongly solvated.

Most of the information which is available pertains to solvation of species in aqueous solutions (Table II), although some information exists regarding the magnitude of solvation energies in other solvents. For example, the free energy of transfer of some ions from water to other solvents:

$$
M^{+}\left(\mathrm{H}_{2} \mathrm{O}\right) \rightarrow M^{+} \text {(Solvent) }
$$

has been determined and typical values for several water-solvent systems are given in Table III. Such data indicate that there is little difference between the energies of solvation of the same species by different solvents. Indeed, some substances, for example methanol, are slightly better solvents for ions than is water. The ability of solvents other than water to stabilize ionic species is borne out by such solvation data as are available for other solvent systems (Table IV). The ions selected for comparison in Table IV are both relatively large $\left(\mathrm{Cs}^{+}=0.169\right.$ $\left.\mathrm{nm}, \mathrm{I}^{-}=0.216 \mathrm{~nm}\right)$ and are probably free of specific solvation

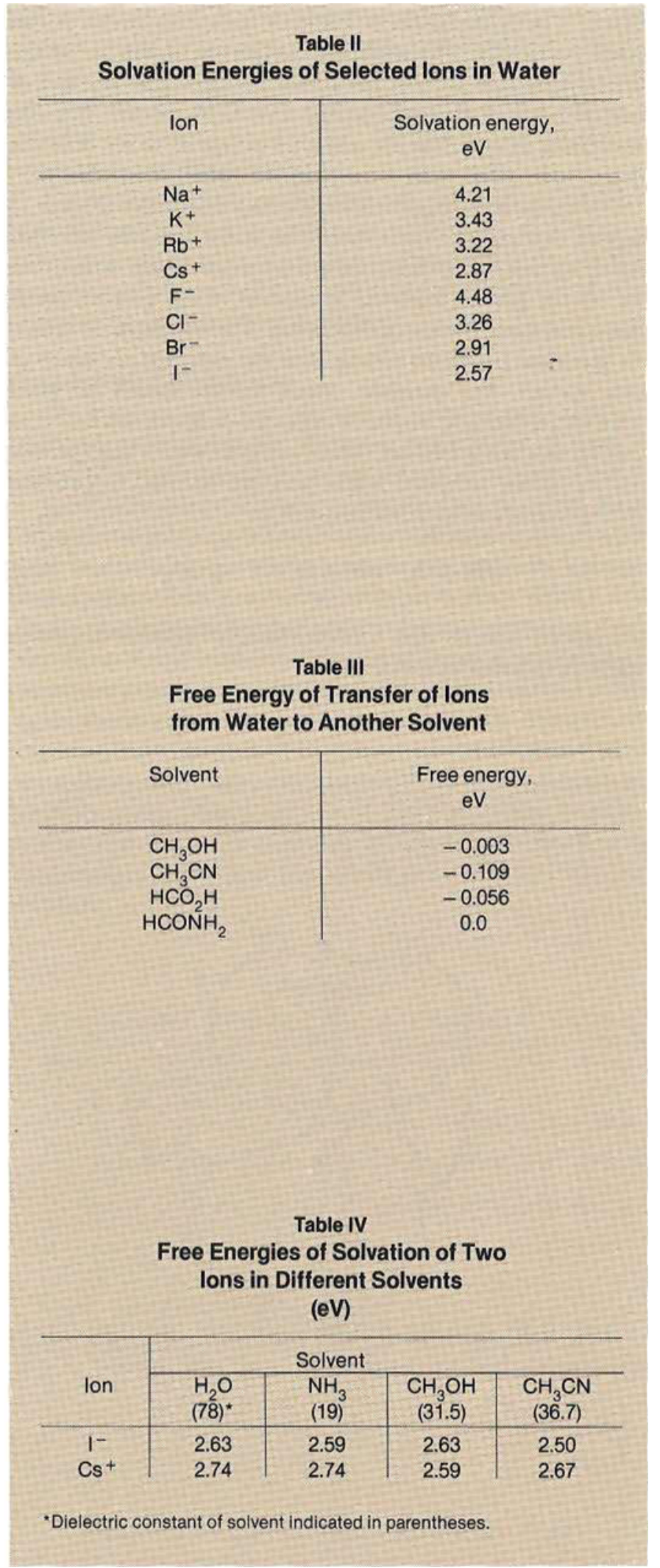


effects. The information in Table IV reinforces several points made previously. The solvation energies of anions are of the same magnitude as those of cations, and solvation energies of ions do not vary greatly from one solvent to another. It is also interesting to note that the solvation energy of an ion does not appear to be affected by the dielectric constant of the solvent.

The qualitative arguments regarding energetics (Figure 1) suggest that metal anions should be stable in many solvents, assuming that the sublimation energy is not so large as to offset the energy gained from the formation of the gaseous metal anion and the solvation of that anion. These arguments ignore the potential problems arising from the expected chemistry of such species. For example, metal anions should be good reducing agents and might be expected to attack solvents that contain oxidizing moities. Thus, solvents' with a high proton activity (acidic solvents) might undergo reaction to produce hydrogen and a solid metal lattice $M_{(S)}$ :

$$
H S+M^{-} \rightarrow 1 / 2 H+S^{-}+M_{(S)}
$$

and aprotic solvents that contain reducible functional groups might be reduced by metal anions:

$$
M^{-}+S \rightarrow M_{(S)}+S^{*}
$$

An additional possible way in which metal anions may be destabilized by a solvent involves the formation of solvated electrons $e^{-}$(Solvent) ${ }_{X}$ :

$$
M^{-}+X S \rightarrow M_{(S)}+e^{-}(\text {Solvent })_{X}
$$

It has been reported (2) that a number of solvents (for example, tetrahydrofuran, ammonia and amines) exhibit the unusual property of forming stable solutions which contain solvated electrons. A part of the driving force for the chemical destabilizing processes (equations (3) to (5)) involves the formation of the solid metal lattice which, indirectly, is associated with the energy of sublimation, as discussed earlier.

The foregoing discussion of the factors which affect metal anion formation helps define, in broad terms, the characteristics of solvents in which metal anions might be expected to exist as stable species. Thus, the solvents should have low proton activity (the 'basic' or 'aprotic' solvent in the usual classification scheme (3)), and they should incorporate neither reducible moities such as the carbonyl function nor, in all probability, any double-bonded units such as $\mathrm{C}=\mathrm{N}$. From this point of view, water is not expected to be a good solvent, but anhydrous ammonia, the amines and perhaps the ethers would be expected to have good solvation properties for metal anions.

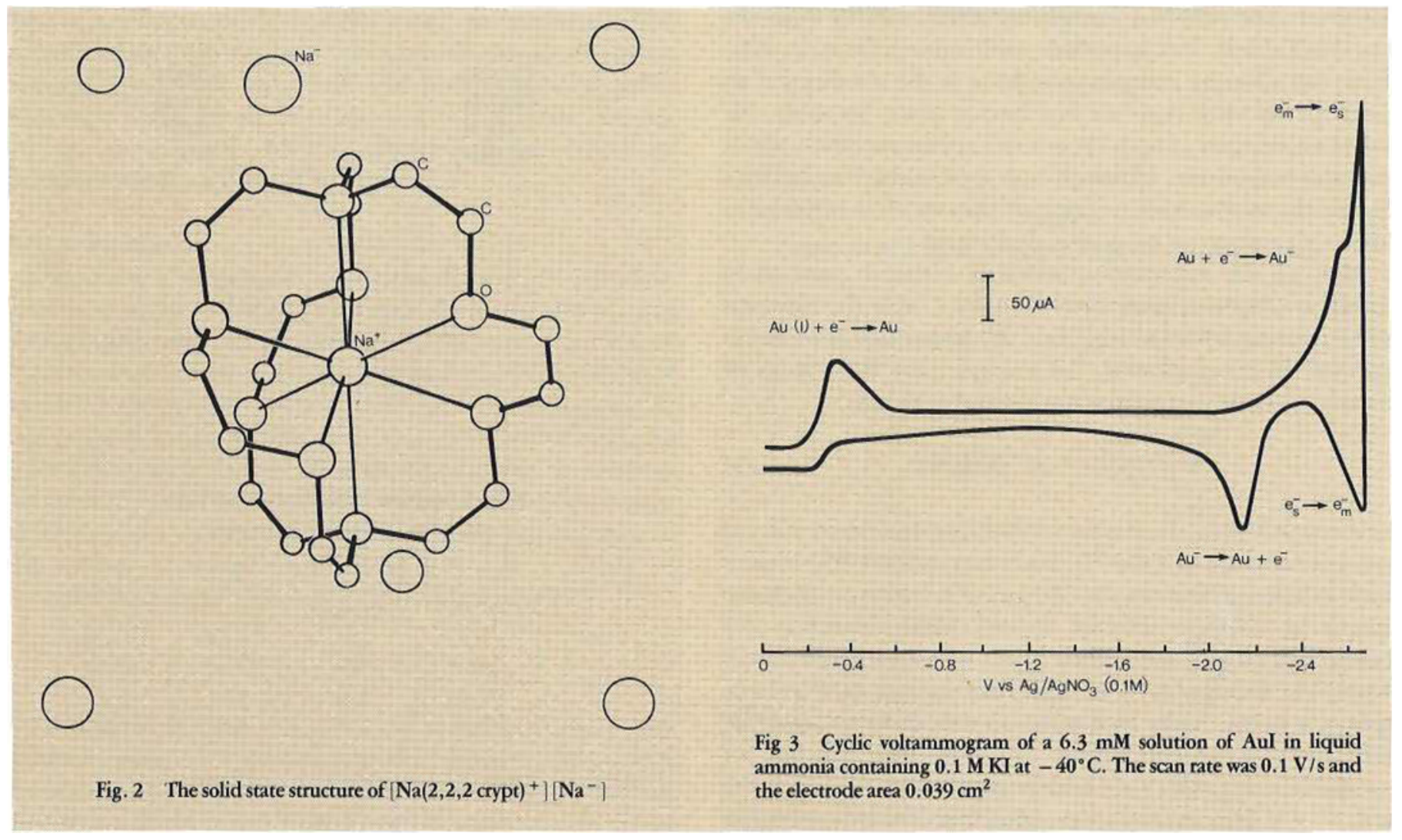




\section{The Existence of Metal Anions}

Historically, the solutions of alkali metals in ammonia were the first systems in which metal anions were characterized. These solutions show properties which vary strikingly with the identity of the alkali metal used. With the exception of that of lithium solutions, the optical spectra show a metal-dependent absorption band in the near infrared region. These bands are assigned to the species $M^{-}(M=\mathrm{Na}, \mathrm{K}, \mathrm{Rb}$ or $\mathrm{Cs})$. The conductivities of solutions that show this metal-dependent band are drastically different from those of solutions which also exhibit the solvated electron band. The chemical reactivity of the metal anion is very different from that of the solvated electron as determined by stopped flow (4) and pulse-radiolyses (5) studies. Finally, a crystalline compound containing the sodium anion, that is $\mathrm{Na}^{+} \mathrm{CNa}^{-}$, where $\mathrm{C}$ is the $2,2,2$ cryptand, has been isolated and its structure determined (Figure 2) (6). The structure shows clearly the presence of complexed $\mathrm{Na}^{+}$as well as the arrangement of $\mathrm{Na}^{-}$in the crystal. Spectroscopic evidence also exists which indicates the existence of solid compounds containing $\mathrm{K}^{-}, \mathrm{Rb}^{-}$and $\mathrm{Cs}^{-}$(7). Electrochemical evidence has been obtained for the reversible formation of $M^{-}$ $(M=\mathrm{Na}, \mathrm{K}, \mathrm{Rb}, \mathrm{Cs})$ in anhydrous ethylenediamine.

\section{Anionic Gold}

The above background information on the existence of alkali metal anions indicates the experimental techniques that can be employed to investigate the presence of other metal anions in non-aqueous solvents, namely electrochemical and spectroscopic analysis. Using these tools, it has been shown in the author's laboratory (8) that the solutions formed when elemental gold is allowed to react with liquid ammonia solutions of solvated electrons contain the $\mathrm{Au}^{-}$species. Thus, metal-ammonia solutions used for dissolving elemental gold exhibit a sharp spectroscopic absorption band at $278 \mathrm{~nm}$, at a temperature of $-65^{\circ} \mathrm{C}$, with an intensity $\left(\mathrm{e}=5 \times 10^{4}\right)$ and a temperature coefficient that are characteristic of charge transfer spectra of other anionic species (9). Electrochemical experiments (Figure 3) show unambiguously (10) the stability of the $\mathrm{Au}^{-}$species in liquid ammonia solutions.

Figure 3 is a typical cyclic voltammogram of an ammonia solution containing gold iodide AuI. The curves show evidence for three oxidation states of gold being present: $\mathrm{Au}^{+}, \mathrm{Au}^{\circ}$ and $\mathrm{Au}^{-}$. Controlled potential electrolysis at $-0.5 \mathrm{~V}$ resulted in the deposition of gold on the platinum working electrode. When the electrode potential was made more negative to allow the generation of solvated electrons $(-2.5 \mathrm{~V})$, the freshly deposited gold was cathodically stripped. The cyclic voltammogram of the resulting solution after excess solvated electrons were removed coulometrically, indicated the presence of an anodic wave at $-2.15 \mathrm{~V}$ corresponding to the oxidation of $\mathrm{Au}^{-} \mathrm{t}$ to $\mathrm{Au}^{\circ}$. The electrolytic oxidation of the solution (at $-2.0 \mathrm{~V}$ ) resulted in deposition of gold on the platinum anode surface. The gold deposit produced in this way was bright and could be polished to a high lustre. Examination of the surface by electron spectroscopy for chemical analysis showed strong bands at binding energies of $82,85,332,350$ and $545 \mathrm{eV}$, which are characteristic of metallic gold.

The author and his colleagues are currently using these electrochemical and spectroscopic techniques as probes to investigate the stability of $\mathrm{Au}$ in a variety of solvents other than liquid ammonia, as well as to investigate the existence of other metal anions in non-aqueous solvents.

\section{Technological Significance}

The existence of stable solutions of metal anions points immediately to technologically interesting possibilities with regard to potential new processes for the separation and/or refining of metals.

Specifically, our preliminary experimental results on the anodic deposition of gold suggest a method of separating and purifying gold from base metals in one relatively simple step, that is by deposition from a suitable solvent containing solvated electrons. While it is difficult to foresee from these preliminary experiments whether anodic deposition of gold would be likely to be of practical use either in the separation process or perhaps even in specialised industrial plating fields, this procedure does avoid the use of cyanide. Moreover, the oxidation of $\mathrm{Au}^{-}$occurs with 100 per cent current efficiency without the evolution of hydrogen which accompaniescathodic deposition in aqueous media. Finally, the use of liquid ammonia provides a unique opportunity of examining the course and nature of the deposition of a metal both cathodically and anodically in the same medium.

\section{References}

1 H. Hotopand W.C. Lineberger, J. Phys. Chem. Ref. Data, 1975, 4, 539-576 2 J.C. Thompson, in 'The Chemistry of Nonaqueous Solvents,' Vol. II, edited by J.J. Lagowski, Academic Press, 1970, p. 265

3 J.J. Lagowski, Rev. Chim. Miner., 1978, 15, 1-22

4 J.L. Dye, Acc. Chem. Res., 1968, 1, 306-312

5 J.L. Dye, M.G. de Bocker, J.A. Eyre and M.L. Dorfman, J. Phys. Chem. $1972,76,839.846$

6 I.J. Tehan, B.L. Barnett and J.L. Dye, J. Am. Chem. Soc., 1974, 96, 7203-7208

7 J.L. Dye, M.R. Yemen, M.G. DaGue and J.-M. Lehn, J. Chem. Pbys., $1978,68,1665-1670$

M.G. DaGue, J.S. Landers, H.L. Lewis and J.L. Dye, Chem. Phys, Lett. $1979,66,169-172$

J.L. Dye, M.G. DaGue, M.R. Yemen, J.S. Landers and H.L. Lewis, J. Pbys. Chem., 1980, 84, 1096-1099

8 W.J. Peer and J.J. Lagowski, J. Am. Chem. Soc. . 1978, 100, 6260-6261

9 M.J. Blandamer and M.F. Fox, Chem:-Rev:; 1970, 70, 59-93

10 T.H. Teherani, W.J. Peer, J.J. Lagowski and A.J. Bard, J. Electrochem. Soc. $1978,125,1717-1718$ 